\title{
NIELSEN NUMBER OF A COVERING MAP
}

\author{
JERZY JEZIERSKI
}

Received 23 November 2004; Revised 13 May 2005; Accepted 24 July 2005

We consider a finite regular covering $p_{H}: \tilde{X}_{H} \rightarrow X$ over a compact polyhedron and a map $f: X \rightarrow X$ admitting a lift $\tilde{f}: \tilde{X}_{H} \rightarrow \widetilde{X}_{H}$. We show some formulae expressing the Nielsen number $N(f)$ as a linear combination of the Nielsen numbers of its lifts.

Copyright () 2006 Jerzy Jezierski. This is an open access article distributed under the Creative Commons Attribution License, which permits unrestricted use, distribution, and reproduction in any medium, provided the original work is properly cited.

\section{Introduction}

Let $X$ be a finite polyhedron and let $H$ be a normal subgroup of $\pi_{1}(X)$. We fix a covering $p_{H}: \tilde{X}_{H} \rightarrow X$ corresponding to the subgroup $H$, that is, $p_{\#}\left(\pi_{1}\left(\tilde{X}_{H}\right)\right)=H$.

We assume moreover that the subgroup $H$ has finite rank, that is, the covering $p_{H}$ is finite. Let $f: X \rightarrow X$ be a map satisfying $f(H) \subset H$. Then $f$ admits a lift

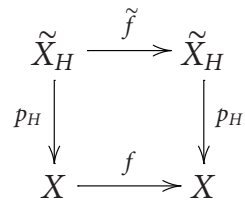

Is it possible to find a formula expressing the Nielsen number $N(f)$ by the numbers $N(\tilde{f})$ where $\tilde{f}$ runs the set of all lifts? Such a formula seems very desirable since the difficulty of computing the Nielsen number often depends on the size of the fundamental group. Since $\pi_{1} \tilde{X} \subset \pi_{1} X$, the computation of $N(\tilde{f})$ may be simpler. We will translate this problem to algebra. The main result of the paper is Theorem 4.2 expressing $N(f)$ as a linear combination of $\left\{N\left(\tilde{f}_{i}\right)\right\}$, where the lifts are representing all the $H$-Reidemeister classes of $f$.

The discussed problem is analogous to the question about "the Nielsen number product formula” raised by Brown in 1967 [1]. A locally trivial fibre bundle $p: E \rightarrow B$ and a 
fibre map $f: E \rightarrow E$ were given and the question was how to express $N(f)$ by $N(\bar{f})$ and $N\left(f_{b}\right)$, where $\bar{f}: B \rightarrow B$ denoted the induced map of the base space and $f_{b}$ was the restriction to the fibre over a fixed point $b \in \operatorname{Fix}(\bar{f})$. This problem was intensively investigated in 70ties and finally solved in 1980 by You [4]. At first sufficient conditions for the "product formula" were formulated: $N(f)=N(\bar{f}) N\left(f_{b}\right)$ assuming that $N\left(f_{b}\right)$ is the same for all fixed points $b \in \operatorname{Fix}(\bar{f})$. Later it turned out that in general it is better to expect the formula

$$
N(f)=N\left(f_{b_{1}}\right)+\cdots+N\left(f_{b_{s}}\right)
$$

where $b_{1}, \ldots, b_{s}$ represent all the Nielsen classes of $\bar{f}$. One may find an analogy between the last formula and the formulae of the present paper. There are also other analogies: in both cases the obstructions to the above equalities lie in the subgroups $\left\{\alpha \in \pi_{1} X ; f_{\#} \alpha=\right.$ $\alpha\} \subset \pi_{1} X$.

\section{Preliminaries}

We recall the basic definitions $[2,3]$. Let $f: X \rightarrow X$ be a self-map of a compact polyhedron. Let $\operatorname{Fix}(f)=\{x \in X ; f(x)=x\}$ denote the fixed point set of $f$. We define the Nielsen relation on $\operatorname{Fix}(f)$ putting $x \sim y$ if there is a path $\omega:[0,1] \rightarrow X$ such that $\omega(0)=x$, $\omega(1)=y$ and the paths $\omega, f \omega$ are fixed end point homotopic. This relation splits the set $\operatorname{Fix}(f)$ into the finite number of classes $\operatorname{Fix}(f)=A_{1} \cup \cdots \cup A_{s}$. A class $A \subset \operatorname{Fix}(f)$ is called essential if its fixed point index $\operatorname{ind}(f ; A) \neq 0$. The number of essential classes is called the Nielsen number and is denoted by $N(f)$. This number has two important properties. It is a homotopy invariant and is the lower bound of the number of fixed points: $N(f) \leq \# \operatorname{Fix}(g)$ for every map $g$ homotopic to $f$.

Similarly we define the Nielsen relation modulo a normal subgroup $H \subset \pi_{1} X$. We assume that the map $f$ preserves the subgroup $H$, that is, $f_{\#} H \subset H$. We say that then $x \sim_{H} y$ if $\omega=f \omega \bmod H$ for a path $\omega$ joining the fixed points $x$ and $y$. This yields $H$-Nielsen classes and H-Nielsen number $N_{H}(f)$. For the details see [4].

Let us notice that each Nielsen class $\bmod H$ splits into the finite sum of ordinary Nielsen classes (i.e., classes modulo the trivial subgroup): $A=A_{1} \cup \cdots \cup A_{s}$. On the other hand $N_{H}(f) \leq N(f)$.

We consider a regular finite covering $p: \tilde{X}_{H} \rightarrow X$ as described above.

Let

$$
\mathrm{O}_{X H}=\left\{\gamma: \tilde{X}_{H} \longrightarrow \tilde{X}_{H} ; p_{H} \gamma=p_{H}\right\}
$$

denote the group of natural transformations of this covering and let

$$
\operatorname{lift}_{H}(f)=\left\{\tilde{f}: \tilde{X}_{H} \longrightarrow \tilde{X}_{H} ; p_{H} \tilde{f}=f p_{H}\right\}
$$

denote the set of all lifts. 
We start by recalling classical results giving the correspondence between the coverings and the fundamental groups of a space.

Lemma 2.1. There is a bijection $\mathrm{O}_{X H}=p_{H}^{-1}\left(x_{0}\right)=\pi_{1}(X) / H$ which can be described as follows:

$$
\gamma \sim \gamma\left(\tilde{x}_{0}\right) \sim p_{H}(\tilde{\gamma})
$$

We fix a point $\tilde{x}_{0} \in p_{H}^{-1}\left(x_{0}\right)$. For a natural transformation $\gamma \in O_{X H}, \gamma\left(\tilde{x}_{0}\right) \in p_{H}^{-1}\left(x_{0}\right)$ is a point and $\tilde{\gamma}$ is a path in $\tilde{X}_{H}$ joining the points $\tilde{x}_{0}$ and $\gamma\left(\tilde{x}_{0}\right)$. The bijection is not canonical. It depends on the choice of $x_{0}$ and $\tilde{x}_{0}$.

Let us notice that for any two lifts $\tilde{f}, \tilde{f}^{\prime} \in \operatorname{lift}_{H}(f)$ there exists a unique $\gamma \in \mathrm{O}_{X H}$ satisfying $\tilde{f}^{\prime}=\gamma \tilde{f}$. More precisely, for a fixed lift $\tilde{f}$, the correspondence

$$
\mathrm{O}_{X H} \ni \alpha \longrightarrow \alpha \tilde{f} \in \operatorname{lift}_{H}(f)
$$

is a bijection. This correspondence is not canonical. It depends on the choice of $\tilde{f}$.

The group $\mathrm{O}_{X H}$ is acting on $\operatorname{lift}_{H}(f)$ by the formula

$$
\alpha \circ \tilde{f}=\alpha \cdot \tilde{f} \cdot \alpha^{-1}
$$

and the orbits of this action are called Reidemeister classes mod $H$ and their set is denoted $\mathscr{R}_{H}(f)$. Then one can easily check [3]

(1) $p_{H}(\operatorname{Fix}(\tilde{f})) \subset \operatorname{Fix}(f)$ is either exactly one $H$-Nielsen class of the map $f$ or is empty (for any $\tilde{f} \in \operatorname{lift}_{H}(f)$ )

(2) $\operatorname{Fix}(f)=\bigcup_{\tilde{f}} p_{H}(\operatorname{Fix}(\tilde{f}))$ where the summation runs the set $\operatorname{lift}_{H}(f)$

(3) if $p_{H}(\operatorname{Fix}(\tilde{f})) \cap p_{H}\left(\operatorname{Fix}\left(\tilde{f}^{\prime}\right)\right) \neq \varnothing$ then $\tilde{f}, \tilde{f}^{\prime}$ represent the same Reidemeister class in $\mathscr{R}_{H}(f)$

(4) if $\tilde{f}, \tilde{f}^{\prime}$ represent the same Reidemeister class then $p_{H}(\operatorname{Fix}(\tilde{f}))=p_{H}\left(\operatorname{Fix}\left(\tilde{f}^{\prime}\right)\right)$.

Thus $\operatorname{Fix}(f)=\bigcup_{\tilde{f}} p_{H}(\operatorname{Fix}(\tilde{f}))$ is the disjoint sum where the summation is over a subset containing exactly one lift $\tilde{f}$ from each $H$-Reidemeister class. This gives the natural inclusion from the set of Nielsen classes modulo $H$ into the set of $H$-Reidemeister classes

$$
\mathcal{N}_{H}(f) \longrightarrow \mathscr{R}_{H}(f)
$$

The $H$-Nielsen class $A$ is sent into the $H$-Reidemeister class represented by a lift $\tilde{f}$ satisfying $p_{H}(\operatorname{Fix}(\tilde{f}))=A$. By (1) and (2) such lift exists, by (3) the definition is correct and (4) implies that this map is injective. 


\section{Lemmas}

For a lift $\tilde{f} \in \operatorname{lift}_{H}(f)$, a fixed point $x_{0} \in \operatorname{Fix}(f)$ and an element $\beta \in \pi_{1}\left(X ; x_{0}\right)$ we define the subgroups

$$
\begin{gathered}
\mathscr{L}(\tilde{f})=\left\{\gamma \in \mathcal{O}_{X H} ; \tilde{f} \gamma=\gamma \tilde{f}\right\} \\
C\left(f_{\#}, x_{0} ; \beta\right)=\left\{\alpha \in \pi_{1}\left(X ; x_{0}\right) ; \alpha \beta=\beta f_{\#}(\alpha)\right\} \\
C_{H}\left(f_{\#}, x_{0} ; \beta\right)=\left\{[\alpha]_{H} \in \pi_{1}\left(X ; x_{0}\right) / H\left(x_{0}\right) ; \alpha \beta=\beta f_{\#}(\alpha) \text { modulo } H\right\} .
\end{gathered}
$$

If $\beta=1$ we will write simply $C\left(f_{\#}, x_{0}\right)$ or $C_{H}\left(f_{\#}, x_{0}\right)$.

We notice that the canonical projection $j: \pi_{1}\left(X ; x_{0}\right) \rightarrow \pi_{1}\left(X ; x_{0}\right) / H\left(x_{0}\right)$ induces the homomorphism $j: C\left(f_{\#}, x_{0} ; \beta\right) \rightarrow C_{H}\left(f_{\#}, x_{0} ; \beta\right)$.

Lemma 3.1. Let $\tilde{f}$ be a lift of $f$ and let $\tilde{A}$ be a Nielsen class of $\tilde{f}$. Then $p_{H}(\tilde{A}) \subset \operatorname{Fix}(f)$ is a Nielsen class of $f$. On the other hand if $A \subset \operatorname{Fix}(f)$ is a Nielsen class of $f$ then $p_{H}^{-1}(A) \cap$ $\operatorname{Fix}(\tilde{f})$ splits into the finite sum of Nielsen classes of $\tilde{f}$.

Proof. It is evident that $p_{H}(\tilde{A})$ is contained in a Nielsen class $A \subset \operatorname{Fix}(f)$. Now we show that $A \subset p_{H}(\tilde{A})$. Let us fix a point $\tilde{x}_{0} \in \tilde{A}$ and let $x_{0}=p_{H}\left(\tilde{x}_{0}\right)$. Let $x_{1} \in A$. We have to show that $x_{1} \in p_{H}(\tilde{A})$. Let $\omega: I \rightarrow X$ establish the Nielsen relation between the points $\omega(0)=x_{0}$ and $\omega(1)=x_{1}$ and let $h(t, s)$ denote the homotopy between $\omega=h(\cdot, 0)$ and $f \omega=h(\cdot, 1)$. Then the path $\omega$ lifts to a path $\widetilde{\omega}: I \rightarrow \tilde{X}_{H}, \widetilde{\omega}(0)=\tilde{x}_{0}$. Let us denote $\widetilde{\omega}(1)=$ $\tilde{x}_{1}$. It remains to show that $\tilde{x}_{1} \in \tilde{A}$. The homotopy $h$ lifts to $\tilde{h}: I \times I \rightarrow \tilde{X}_{H}, \tilde{h}(0, s)=\tilde{x}_{0}$. Then the paths $\tilde{h}(\cdot, 1)$ and $\tilde{f} \tilde{\omega}$ as the lifts of $f \omega$ starting from $\tilde{x}_{0}$ are equal. Now $\tilde{f}\left(\tilde{x}_{1}\right)=$ $\tilde{f}(\tilde{\omega}(1))=\tilde{h}(1,1)=\tilde{h}(1,0)=\tilde{\omega}(1)=\tilde{x}_{1}$. Thus $\tilde{x}_{1} \in \operatorname{Fix}(\tilde{f})$ and the homotopy $\tilde{h}$ gives the Nielsen relation between $\tilde{x}_{0}$ and $\tilde{x}_{1}$ hence $\tilde{x}_{1} \in \tilde{A}$.

Now the second part of the lemma is obvious.

Lemma 3.2. Let $\tilde{A} \subset \operatorname{Fix}(\tilde{f})$ be a Nielsen class of $\tilde{f}$. Let us denote $A=p_{H}(\tilde{A})$. Then

(1) $p_{H}: \widetilde{A} \rightarrow A$ is a covering where the fibre is in bijection with the image $j_{\#}\left(C\left(f_{\#}, x\right)\right) \subset$ $\pi_{1}(X ; x) / H(x)$ for $x \in A$,

(2) the cardinality of the fibre (i.e., $\left.\#\left(p_{H}^{-1}(x) \cap \widetilde{A}\right)\right)$ does not depend on $x \in A$ and we will denote it by $J_{A}$,

(3) if $\tilde{A}^{\prime}$ is another Nielsen class of $\tilde{f}$ satisfying $p_{H}\left(\tilde{A}^{\prime}\right)=p_{H}(\tilde{A})$ then the cardinalities of $p_{H}^{-1}(x) \cap \tilde{A}$ and $p_{H}^{-1}(x) \cap \tilde{A}^{\prime}$ are the same for each point $x \in A$.

Proof. (1) Since $p_{H}$ is a local homeomorphism, the projection $p_{H}: \widetilde{A} \rightarrow A$ is the covering.

(2) We will show a bijection $\phi: j\left(C\left(f_{\#} ; x_{0}\right)\right) \rightarrow p_{H}^{-1}\left(x_{0}\right) \cap \tilde{A}$ (for a fixed point $x_{0} \in A$ ).

Let $\alpha \in C\left(f_{\#}\right)$. Let us fix a point $\tilde{x}_{0} \in p_{H}^{-1}\left(x_{0}\right)$. Let $\tilde{\alpha}: I \rightarrow \tilde{X}$ denote the lift of $\alpha$ starting from $\tilde{\alpha}(0)=\tilde{x}_{0}$. We define $\phi\left([\alpha]_{H}\right)=\tilde{\alpha}(1)$. We show that

(2a) The definition is correct. Let $[\alpha]_{H}=\left[\alpha^{\prime}\right]_{H}$. Then $\alpha \equiv \alpha^{\prime} \bmod H$ hence $\tilde{\alpha}(1)=$ $\tilde{\alpha}^{\prime}(1)$. Now we show that $\tilde{\alpha}(1) \in \widetilde{A}$. Since $\alpha \in C\left(f_{\#}\right)$, there exists a homotopy $h$ between the loops $h(\cdot, 0)=\alpha$ and $h(\cdot, 1)=f \alpha$. The homotopy lifts to $\tilde{h}: I \times I \rightarrow \tilde{X}_{H}, \tilde{h}(0, s)=\tilde{x}_{0}$. Then $\tilde{x}_{1}=\tilde{h}(1, s)$ is also a fixed point of $\tilde{f}$ and moreover $\tilde{h}$ is the homotopy between the paths $\tilde{\omega}$ and $\tilde{f} \tilde{\omega}$. Thus $\tilde{x}_{0}, \tilde{x}_{1} \in \operatorname{Fix}(\tilde{f})$ are Nielsen related hence $\tilde{x}_{1} \in \tilde{A}$. 
(2b) $\phi$ is onto. Let $\tilde{x}_{1} \in p_{H}^{-1}\left(x_{0}\right) \cap \tilde{A}$. Now $\tilde{x}_{0}, \tilde{x}_{1} \in \operatorname{Fix}(\tilde{f})$ are Nielsen related. Let $\tilde{\omega}$ : $I \rightarrow \tilde{X}_{H}$ establish this relation $(\tilde{f} \tilde{\omega} \sim \tilde{\omega})$. Now

$$
f\left(p_{H} \tilde{\omega}\right)=p_{H} \tilde{f} \tilde{\omega} \sim p_{H} \tilde{\omega}
$$

hence $p_{H} \tilde{\omega} \in C\left(f_{\#} ; x_{0}\right)$. Moreover $\phi\left[p_{H} \tilde{\omega}\right]_{H}=\widetilde{\omega}(1)=\tilde{x}_{1}$.

(2c) $\phi$ is injective. Let $[\alpha]_{H},\left[\alpha^{\prime}\right]_{H} \in j\left(C\left(f_{\#}\right)\right)$ and let $\tilde{\alpha}, \tilde{\alpha}^{\prime}: I \rightarrow \tilde{X}_{H}$ be their lifts starting from $\tilde{\alpha}(0)=\tilde{\alpha}^{\prime}(0)=\tilde{x}_{0}$. Suppose that $\phi[\alpha]_{H}=\phi\left[\alpha^{\prime}\right]_{H}$. This means $\tilde{\alpha}(1)=\tilde{\alpha}^{\prime}(1) \in \tilde{X}_{H}$. Thus $p_{H}\left(\tilde{\alpha} * \tilde{\alpha}^{\prime-1}\right)=\alpha * \alpha^{\prime-1} \in H$ which implies $[\alpha]_{H}=\left[\alpha^{\prime}\right]_{H}$.

(3) Let $x_{0} \in p_{H}(\widetilde{A})=p_{H}\left(\tilde{A}^{\prime}\right)$. Then by the above $\#\left(p^{-1}\left(x_{0}\right) \cap \widetilde{A}\right)=j_{\#}\left(C\left(f_{\#}\right)\right)=$ $\#\left(p^{-1}\left(x_{0}\right) \cap \widetilde{A}^{\prime}\right)$.

Lemma 3.3. The restriction of the covering map $p_{H}: \operatorname{Fix}(\tilde{f}) \rightarrow p_{H}(\operatorname{Fix}(\tilde{f}))$ is a covering. The fibre over each point is in a bijection with the set

$$
\mathscr{L}(\tilde{f})=\left\{\gamma \in \mathcal{O}_{X H} ; \tilde{f} \gamma=\gamma \tilde{f}\right\}
$$

Proof. Since the fibre of the covering $p_{H}$ is discrete, the restriction $p_{H}: \operatorname{Fix}(\tilde{f}) \rightarrow$ $p_{H}(\operatorname{Fix}(\tilde{f}))$ is a locally trivial bundle. Let us fix points $x_{0} \in p_{H}(\operatorname{Fix}(\tilde{f})), \tilde{x}_{0} \in p_{H}^{-1}\left(x_{0}\right) \cap$ $\operatorname{Fix}(\tilde{f})$. We recall that

$$
\alpha: p_{H}^{-1}\left(x_{0}\right) \longrightarrow \mathrm{O}_{X H}
$$

where $\alpha_{\tilde{x}} \in \mathcal{O}_{X H}$ is characterized by $\alpha_{\tilde{x}}\left(\tilde{x}_{0}\right)=\tilde{x}$, is a bijection. We will show that $\alpha\left(p_{H}^{-1}\left(x_{0}\right) \cap \operatorname{Fix}(\tilde{f})\right)=\mathscr{L}(\tilde{f})$.

Let $\tilde{f}(\tilde{x})=\tilde{x}$ for an $\tilde{x} \in p_{H}^{-1}\left(x_{0}\right)$. Then

$$
\tilde{f} \alpha_{\tilde{x}}\left(\tilde{x}_{0}\right)=\tilde{f}(\tilde{x})=\tilde{x}=\alpha_{\tilde{x}}\left(\tilde{x}_{0}\right)=\alpha_{\tilde{x}} \tilde{f}\left(\tilde{x}_{0}\right)
$$

which implies $\tilde{f} \alpha_{\tilde{x}}=\alpha_{\tilde{x}} \tilde{f}$ hence $\alpha_{\tilde{x}} \in \mathscr{L}(\tilde{f})$.

Now we assume that $\tilde{f} \alpha_{\tilde{x}}=\alpha_{\tilde{x}} \tilde{f}$. Then in particular $\tilde{f} \alpha_{\tilde{x}}\left(\tilde{x}_{0}\right)=\alpha_{\tilde{x}} \tilde{f}\left(\tilde{x}_{0}\right)$ which gives $\tilde{f}(\tilde{x})=\alpha_{\tilde{x}}\left(\tilde{x}_{0}\right), \tilde{f}(\tilde{x})=\tilde{x}$ hence $\tilde{x} \in \operatorname{Fix}(\tilde{f})$.

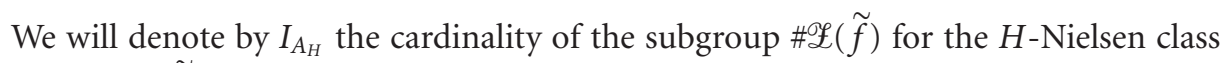
$A_{H}=p_{H}(\operatorname{Fix}(\tilde{f}))$. We will also write $I_{A_{i}}=I_{A_{H}}$ for any Nielsen class $A_{i}$ of $f$ contained in A.

Lemma 3.4. Let $A_{0} \subset \operatorname{Fix}(f)$ be a Nielsen class and let $\tilde{A}_{0} \subset \operatorname{Fix}(\tilde{f})$ be a Nielsen class contained in $p_{H}^{-1}\left(A_{0}\right)$. Then, by Lemma $3.1 A_{0}=p_{H}\left(\widetilde{A}_{0}\right)$ and moreover

$$
\begin{gathered}
\operatorname{ind}\left(\tilde{f} ; p_{H}^{-1}\left(A_{0}\right)\right)=I_{A_{0}} \cdot \operatorname{ind}\left(f ; A_{0}\right) \\
\quad \operatorname{ind}\left(\tilde{f} ; \tilde{A}_{0}\right)=J_{A_{0}} \cdot \operatorname{ind}\left(f ; A_{0}\right) .
\end{gathered}
$$


Proof. Since the index is the homotopy invariant we may assume that $\operatorname{Fix}(f)$ is finite. Now for any fixed points $x_{0} \in \operatorname{Fix}(f), \tilde{x}_{0} \in \operatorname{Fix}(\tilde{f})$ satisfying $p_{H}\left(\tilde{x}_{0}\right)=x_{0}$ we have ind $\left(\tilde{f}_{0} ; \tilde{x}_{0}\right)=$ $\operatorname{ind}\left(f_{0} ; x_{0}\right)$ since the projection $p_{H}$ is a local homeomorphism. Thus

$$
\text { ind } \begin{aligned}
\left(\tilde{f} ; p_{H}^{-1}\left(A_{0}\right)\right) & =\sum_{x \in A_{0}} \operatorname{ind}\left(\tilde{f} ; p_{H}^{-1}(x)\right)=\sum_{x \in A_{0}} I_{A_{0}} \cdot \operatorname{ind}(f ; x) \\
& =I_{A_{0}} \sum_{x \in A_{0}} \operatorname{ind}(f ; x)=I_{A_{0}} \cdot \operatorname{ind}\left(f ; A_{0}\right) .
\end{aligned}
$$

Similarly we prove the second equality:

$$
\begin{aligned}
\operatorname{ind}\left(\tilde{f} ; \tilde{A}_{0}\right) & =\sum_{x \in A_{0}} \operatorname{ind}\left(\tilde{f} ; p_{H}^{-1}(x) \cap \tilde{A}_{0}\right)=\sum_{x \in A_{0}} \sum_{\tilde{x} \in p_{H}^{-1}(x) \cap \tilde{A}_{0}} \operatorname{ind}(\tilde{f} ; \tilde{x}) \\
& =\sum_{x \in A_{0}} J_{A_{0}} \cdot \operatorname{ind}(f ; x)=J_{A_{0}} \cdot\left(\sum_{x \in A_{0}} \operatorname{ind}(f ; x)\right)=J_{A_{0}} \cdot \operatorname{ind}\left(f ; A_{0}\right) .
\end{aligned}
$$

To get a formula expressing $N(f)$ by the numbers $N(\tilde{f})$ we will need the assumption that the numbers $J_{A}=J_{A^{\prime}}$ for any two $H$-Nielsen related classes $A, A^{\prime} \subset \operatorname{Fix}(f)$. The next lemma gives a sufficient condition for such equality.

LemmA 3.5. Let $x_{0} \in p(\operatorname{Fix}(\tilde{f}))$. If the subgroups $H\left(x_{0}\right), C\left(f, x_{0}\right) \subset \pi_{1}\left(X, x_{0}\right)$ commute, that is, $h \cdot \alpha=\alpha \cdot h$, for any $h \in H\left(x_{0}\right), \alpha \in C\left(f, x_{0}\right)$, then $J_{A}=J_{A^{\prime}}$ for all Nielsen classes $A, A^{\prime} \subset p(\operatorname{Fix}(\tilde{f}))$.

Proof. Let $x_{1} \in p(\operatorname{Fix}(\tilde{f}))$ be another point. The points $x_{0}, x_{1} \in p(\operatorname{Fix}(\tilde{f}))$ are $H$-Nielsen related, that is, there is a path $\omega:[0,1] \rightarrow X$ satisfying $\omega(0)=x_{0}, \omega(1)=x_{1}$ such that $\omega * f\left(\omega^{-1}\right) \in H\left(x_{0}\right)$. We will show that the conjugation

$$
\pi_{1}\left(X, x_{0}\right) \ni \alpha \longrightarrow \omega^{-1} * \alpha * \omega \in \pi_{1}\left(X, x_{1}\right)
$$

sends $C\left(f, x_{0}\right)$ onto $C\left(f, x_{1}\right)$. Let $\alpha \in C\left(f, x_{0}\right)$. We will show that $\omega^{-1} * \alpha * \omega \in C\left(f, x_{1}\right)$. In fact $f\left(\omega^{-1} * \alpha * \omega\right)=\omega^{-1} * \alpha * \omega \Leftrightarrow\left(\omega * f \omega^{-1}\right) * \alpha=\alpha *\left(\omega * f \omega^{-1}\right)$ but the last equality holds since $\omega * f \omega^{-1} \in H\left(x_{0}\right)$ and $\alpha \in C\left(f, x_{0}\right)$.

Remark 3.6. The assumption of the above lemma is satisfied if at least one of the groups $H\left(x_{0}\right), C\left(f, x_{0}\right)$ belongs to the center of $\pi_{1}\left(X ; x_{0}\right)$.

Remark 3.7. Let us notice that if the subgroups $H\left(x_{0}\right), C\left(f, x_{0}\right) \subset \pi_{1}\left(X, x_{0}\right)$ commute then so do the corresponding subgroups at any other point $x_{1} \in p_{H}(\operatorname{Fix}(\tilde{f}))$.

Proof. Let us fix a path $\omega:[0,1] \rightarrow X$. We will show that the conjugation

$$
\pi_{1}\left(X, x_{0}\right) \ni \alpha \longrightarrow \omega^{-1} * \alpha * \omega \in \pi_{1}\left(X, x_{1}\right)
$$

sends $C\left(f, x_{0}\right)$ onto $C\left(f, x_{1}\right)$. Let $\alpha \in C\left(f, x_{0}\right)$. We will show that $\omega^{-1} * \alpha * \omega \in C\left(f, x_{1}\right)$. But the last means $f\left(\omega^{-1} * \alpha * \omega\right)=\omega^{-1} * \alpha * \omega$ hence $f\left(\omega^{-1}\right) * f \alpha * f \omega=\omega^{-1} * \alpha *$ $\omega \Leftrightarrow f\left(\omega^{-1}\right) * \alpha * f \omega=\omega^{-1} * \alpha * \omega \Leftrightarrow\left(\omega * f \omega^{-1}\right) * \alpha=\alpha *\left(\omega * f \omega^{-1}\right)$ and the last 
holds since $\left(\omega * f \omega^{-1}\right) \in H\left(x_{0}\right)$ and $\alpha \in C\left(f, x_{0}\right)$. Now it remains to notice that the elements of $H\left(x_{1}\right), C\left(f ; x_{1}\right)$ are of the form $\omega^{-1} * \gamma * \omega$ and $\omega^{-1} * \alpha * \omega$ respectively for some $\gamma \in H\left(x_{0}\right)$ and $\alpha \in C\left(f, x_{0}\right)$.

Now we will express the numbers $I_{A}, J_{A}$ in terms of the homotopy group homomorphism $f_{\#}: \pi_{1}\left(X, x_{0}\right) \rightarrow \pi_{1}\left(X, x_{0}\right)$ for a fixed point $x_{0} \in \operatorname{Fix}(f)$. Let $\tilde{f}: \widetilde{X}_{H} \rightarrow \tilde{X}_{H}$ be a lift satisfying $\tilde{x}_{0} \in p_{H}^{-1}\left(x_{0}\right) \cap \operatorname{Fix}(\tilde{f})$. We also fix the isomorphism

$$
\pi_{1}\left(X ; x_{0}\right) / H\left(x_{0}\right) \ni \alpha \longrightarrow \gamma_{\alpha} \in \mathrm{O}_{X H}
$$

where $\gamma_{\alpha}\left(\tilde{x}_{0}\right)=\tilde{\alpha}(1)$ and $\tilde{\alpha}$ denotes the lift of $\alpha$ starting from $\tilde{\alpha}(0)=\tilde{x}_{0}$.

We will describe the subgroup corresponding to $C(\tilde{f})$ by this isomorphism and then we will do the same for the other lifts $\tilde{f}^{\prime} \in \operatorname{lift}_{H}(f)$.

LEMMA 3.8.

$$
\tilde{f} \gamma_{\alpha}=\gamma_{f \alpha} \tilde{f}
$$

Proof.

$$
\tilde{f} \gamma_{\alpha}\left(\tilde{x}_{0}\right)=\tilde{f} \tilde{\alpha}(1)=\gamma_{f \alpha}\left(\tilde{x}_{0}\right)=\gamma_{f \alpha} \tilde{f}\left(\tilde{x}_{0}\right)
$$

where the middle equality holds since $\tilde{f} \tilde{\alpha}$ is a lift of the path $f \alpha$ from the point $\tilde{x}_{0}$.

Corollary 3.9. There is a bijection between

$$
\begin{gathered}
\mathscr{L}(\tilde{f})=\left\{\gamma \in O_{X H} ; \tilde{f} \gamma=\gamma \tilde{f}\right\}, \\
C_{H}(f)=\left\{\alpha \in \pi_{1}\left(X ; x_{0}\right) / H\left(x_{0}\right) ; f_{H \#}(\alpha)=\alpha\right\} .
\end{gathered}
$$

Thus

$$
I_{A} / J_{A}=\# \mathscr{L}(\tilde{f}) / \# j(C(f))=\#\left(C_{H}(f) / j(C(f))\right) .
$$

Let us emphasize that $C(f), C_{H}(f)$ are the subgroups of $\pi_{1}\left(X ; x_{0}\right)$ or $\pi_{1}\left(X ; x_{0}\right) / H\left(x_{0}\right)$ respectively where the base point is the chosen fixed point. Now will take another fixed point $x_{1} \in \operatorname{Fix}(f)$ and we will denote $C^{\prime}(f)=\left\{\alpha^{\prime} \in \pi_{1}\left(X ; x_{1}\right) ; f_{\#} \alpha=\alpha\right\}$ and similarly we define $C_{H}^{\prime}(f)$. We will express the cardinality of these subgroups in terms of the group $\pi_{1}\left(X ; x_{0}\right)$.

Lemma 3.10. Let $\eta:[0,1] \rightarrow X$ be a path from $x_{0}$ to $x_{1}$. This path gives rise to the isomorphism $P_{\eta}: \pi_{1}\left(X ; x_{1}\right) \rightarrow \pi_{1}\left(X ; x_{0}\right)$ by the formula $P_{\eta}(\alpha)=\eta \alpha \eta^{-1}$. Let $\delta=\eta \cdot(f \eta)^{-1}$. Then

$$
\begin{gathered}
P_{\eta}\left(C^{\prime}(f)\right)=\left\{\alpha \in \pi_{1}\left(X ; x_{0}\right) ; \alpha \delta=\delta f_{\#}(\alpha)\right\} \\
P_{\eta}\left(C_{H}^{\prime}(f)\right)=\left\{[\alpha] \in \pi_{1}\left(X ; x_{0}\right) / H\left(x_{0}\right) ; \alpha \delta=\delta f_{\#}(\alpha) \text { modulo } H\right\} .
\end{gathered}
$$


Proof. We notice that $\delta$ is a loop based at $x_{0}$ representing the Reidemeister class of the point $x_{1}$ in $\mathscr{R}(f)=\pi_{1}\left(X ; x_{0}\right) / \mathscr{R}$.

We will denote the right-hand side of the above equalities by $C(f ; \delta)$ and $C_{H}(f ; \delta)$ respectively. Let $\alpha^{\prime} \in \pi_{1}\left(X ; x_{1}\right)$. We denote $\alpha=P_{\eta}\left(\alpha^{\prime}\right)=\eta \alpha^{\prime} \eta^{-1}$. We will show that $\alpha \in$ $C(f ; \delta) \Leftrightarrow \alpha^{\prime} \in C^{\prime}(f)$.

In fact $\alpha \in C(f ; \delta) \Leftrightarrow \alpha \delta=\delta \cdot f \alpha \Leftrightarrow\left(\eta \alpha^{\prime} \eta^{-1}\right)\left(\eta \cdot f \eta^{-1}\right)=\left(\eta \cdot f \eta^{-1}\right)\left(f \eta \cdot f \alpha^{\prime}\right.$. $\left.(f \eta)^{-1}\right) \Leftrightarrow \eta \alpha^{\prime} \cdot(f \eta)^{-1}=\eta \cdot f \alpha^{\prime} \cdot(f \eta)^{-1} \Leftrightarrow \alpha^{\prime}=f \alpha^{\prime}$.

Similarly we prove the second equality.

Thus we get the following formulae for the numbers $I_{A}, J_{A}$.

Corollary 3.11. Let $\delta \in \pi_{1}\left(X ; x_{0}\right)$ represent the Reidemeister class $A \in \mathscr{R}(f)$. Then $I_{A}=$ $\# C_{H}(f ; j(\delta)), J_{A}=\# j(C(f ; \delta))$.

\section{Main theorem}

Lemma 4.1. Let $A \subset p_{H}(\operatorname{Fix}(\tilde{f}))$ be a Nielsen class of $f$. Then $p_{H}^{-1} A$ contains exactly $I_{A} / J_{A}$ fixed point classes of $\tilde{f}$.

Proof. Since the projection of each Nielsen class $\tilde{A} \subset p_{H}^{-1}(A) \cap \operatorname{Fix}(\tilde{f})$ is onto $A$ (Lemma $3.1)$, it is enough to check how many Nielsen classes of $\tilde{f}$ cut $p_{H}^{-1}(a)$ for a fixed point $a \in A$. But by Lemma $3.3 p_{H}^{-1}(a) \cap \operatorname{Fix}(\tilde{f})$ contains $I_{A}$ points and by Lemma 3.2 each class in this set has exactly $J_{A}$ common points with $p_{H}^{-1}(a)$. Thus exactly $I_{A} / J_{A}$ Nielsen classes of $\tilde{f}$ are cutting $p_{H}^{-1}(a) \cap \operatorname{Fix}(\tilde{f})$.

Let $f: X \rightarrow X$ be a self-map of a compact polyhedron admitting a lift $\tilde{f}: \tilde{X}_{H} \rightarrow \tilde{X}_{H}$. We will need the following auxiliary assumption:

for any Nielsen classes $A, A^{\prime} \in \operatorname{Fix}(f)$ representing the same class modulo the subgroup $H$ the numbers $J_{A}=J_{A^{\prime}}$.

We fix lifts $\tilde{f}_{1}, \ldots, \tilde{f}_{s}$ representing all $H$-Nielsen classes of $f$, that is,

$$
\operatorname{Fix}(f)=p_{H}\left(\operatorname{Fix}\left(\tilde{f}_{1}\right)\right) \cup \cdots \cup p_{H}\left(\operatorname{Fix}\left(\tilde{f}_{s}\right)\right)
$$

is the mutually disjoint sum. Let $I_{i}, J_{i}$ denote the numbers corresponding to a (Nielsen class of $f) A \subset p_{H}\left(\operatorname{Fix}\left(\tilde{f}_{i}\right)\right)$. By the remark after Lemma 3.3 and by the above assumption these numbers do not depend on the choice of the class $A \subset p_{H}\left(\operatorname{Fix}\left(\tilde{f}_{i}\right)\right)$. We also notice that Lemmas 3.3, 3.2 imply

$$
\begin{gathered}
I_{i}=\# \mathscr{L}\left(\tilde{f}_{i}\right)=\#\left\{\gamma \in O_{X H} ; \gamma \tilde{f}_{i}=\tilde{f}_{i} \gamma\right\} \\
J_{i}=\# j\left(C\left(f_{\#} ; x\right)\right)=\# j\left(\left\{\gamma \in \pi_{1}\left(X, x_{i}\right) ; f_{\#} \gamma=\gamma\right\}\right)
\end{gathered}
$$

for an $x_{i} \in A_{i}$. 
TheOREM 4.2. Let $X$ be a compact polyhedron, $P_{H}: \tilde{X}_{H} \rightarrow \tilde{X}$ a finite regular covering and let $f: X \rightarrow X$ be a self-map admitting a lift $\tilde{f}: \tilde{X}_{H} \rightarrow \tilde{X}_{H}$. We assume that for each two Nielsen classes $A, A^{\prime} \subset \operatorname{Fix}(f)$, which represent the same Nielsen class modulo the subgroup $H$, the numbers $J_{A}=J_{A^{\prime}}$. Then

$$
N(f)=\sum_{i=1}^{s}\left(J_{i} / I_{i}\right) \cdot N\left(\tilde{f}_{i}\right),
$$

where $I_{i}, J_{i}$ denote the numbers defined above and the lifts $\tilde{f}_{i}$ represent all H-Reidemeister classes of $f$, corresponding to nonempty H-Nielsen classes.

Proof. Let us denote $A_{i}=p_{H}\left(\operatorname{Fix}\left(\tilde{f}_{i}\right)\right)$. Then $A_{i}$ is the disjoint sum of Nielsen classes of $f$. Let us fix one of them $A \subset A_{i}$. By Lemma 3.1 $p_{H}^{-1} A \cap \operatorname{Fix}\left(\tilde{f}_{i}\right)$ splits into $I_{A} / J_{A}$ Nielsen classes in $\operatorname{Fix}\left(\tilde{f}_{i}\right)$. By Lemma 3.4 A is essential iff one (hence all) Nielsen classes in $p_{H}^{-1} A \subset$ Fix $\tilde{f}_{i}$ is essential. Summing over all essential classes of $\tilde{f}$ in $A_{i}=p_{A}\left(\operatorname{Fix}\left(\tilde{f}_{i}\right)\right)$ we get

the number of essential Nielsen classes of $f$ in $A_{i}$

$$
=\sum_{A}\left(J_{A} / I_{A}\right) \cdot\left(\text { number of essential Nielsen classes of } \tilde{f}_{i} \text { in } p_{H}^{-1} A\right) \text {, }
$$

where the summation runs the set of all essential Nielsen classes contained in $A_{i}$.

But $J_{A}=J_{i}, I_{A}=I_{i}$ for all $A \subset A_{i}$ hence

$$
\text { (the number of essential Nielsen classes of } \left.f \text { in } A_{i}\right)=J_{i} / I_{i} \cdot N\left(\tilde{f}_{i}\right) \text {. }
$$

Summing over all lifts $\left\{\tilde{f}_{i}\right\}$ representing non-empty $H$-Nielsen classes of $f$ we get

$$
N(f)=\sum_{i}\left(J_{i} / I_{i}\right) \cdot N\left(\tilde{f}_{i}\right)
$$

since $N(f)$ equals the number of essential Nielsen classes in $\operatorname{Fix}(f)=\bigcup_{i=1}^{s} p_{H} \operatorname{Fix}\left(\tilde{f}_{i}\right)$.

Corollary 4.3. If moreover, under the assumptions of Theorem 4.2, $C=J_{i} / I_{i}$ does not depend on $i$ then

$$
N(f)=C \cdot \sum_{i=1}^{s} N\left(\tilde{f}_{i}\right) .
$$

\section{Examples}

In all examples given below the auxiliary assumption $J_{A}=J_{A^{\prime}}$ holds, since the assumptions of Lemma 3.5 are satisfied (in 1,2, 3 and 5 the fundamental groups are commutative and in 4 the subgroup $C\left(f, x_{0}\right)$ is trivial $)$. 
(1) If $\pi_{1} X$ is finite and $p: \tilde{X} \rightarrow X$ is the universal covering (i.e., $H=0$ ) then $\tilde{X}$ is simply connected hence for any lift $\tilde{f}: \tilde{X} \rightarrow \tilde{X}$

$$
N(\tilde{f})= \begin{cases}1 & \text { for } L(\tilde{f}) \neq 0 \\ 0 & \text { for } L(\tilde{f})=0 .\end{cases}
$$

But $L(\tilde{f}) \neq 0$ if and only if the Nielsen class $p(\operatorname{Fix}(\tilde{f})) \subset \operatorname{Fix}(f)$ is essential (Lemma 3.4). Thus

$$
N(f)=\text { number of essential classes }=N\left(\tilde{f}_{1}\right)+\cdots+N\left(\tilde{f}_{s}\right)
$$

where the lifts $\tilde{f}_{1}, \ldots, \tilde{f}_{s}$ represent all Reidemeister classes of $f$.

(2) Consider the commutative diagram

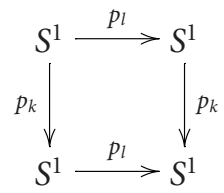

Where $p_{k}(z)=z^{k}, p_{l}(z)=z^{l}, k, l \geq 2$. The map $p_{k}$ is regarded as $k$-fold regular covering map. Then each natural transformation map of this covering is of the form $\alpha(z)=$ $\exp (2 \pi p / k) \cdot z$ for $p=0, \ldots, k-1$ hence is homotopic to the identity map. Now all the lifts of the map $p_{l}$ are maps of degree $l$ hence their Nielsen numbers equal $l-1$. On the other hand the Reidemeister relation of the map $p_{l}: S^{1} \rightarrow S^{1}$ modulo the subgroup $H=\operatorname{im} p_{k \#}$ is given by

$$
\begin{aligned}
\alpha \sim \beta & \Longleftrightarrow \beta=\alpha+p(l-1) \in k \cdot \mathbb{Z} \quad \text { for a } p \in \mathbb{Z} \\
& \Longleftrightarrow \beta=\alpha+p(l-1)+q k \quad \text { for some } p, q \in \mathbb{Z} \\
& \Longleftrightarrow \alpha=\beta \text { modulo g.c.d. }(l-1, k) .
\end{aligned}
$$

Thus $\# \mathscr{S}_{H}\left(p_{l}\right)=$ g.c.d. $(l-1, k)$. Now the sum

$$
\sum_{p_{l}^{\prime}} N\left(p_{l}^{\prime}\right)=(\text { g.c.d. }(l-1, k)) \cdot(l-1),
$$

(where the summation runs the set having exactly one common element with each $H$ Reidemeister class) equals $N\left(p_{l}\right)=l-1$ iff the numbers $k, l-1$ are relatively prime.

Notice that in our notation $I=$ g.c.d. $(l-1, k)$ while $J=1$.

(3) Let us consider the action of the cyclic group $\mathbb{Z}_{8}$ on $S^{3}=\left\{\left(z, z^{\prime}\right) \in \mathbb{C} \times \mathbb{C} ;|z|^{2}+\right.$ $\left.\left|z^{\prime}\right|^{2}=1\right\}$ given by the cyclic homeomorphism

$$
S^{3} \ni\left(z, z^{\prime}\right) \longrightarrow\left(\exp (2 \pi i / 8) \cdot z, \exp (2 \pi i / 8) \cdot z^{\prime}\right) \in S^{3} .
$$

The quotient space is the lens space which we will denote $L_{8}$. We will also consider the quotient space of $S^{3}$ by the action of the subgroup $2 \mathbb{Z}_{4} \subset \mathbb{Z}_{8}$. Now the quotient group is 
also a lens space which we will denote by $L_{4}$. Let us notice that there is a natural 2 -fold covering $p_{H}: L_{4} \rightarrow L_{8}$

$$
L_{4}=S^{3} / \mathbb{Z}_{4} \ni\left[z, z^{\prime}\right] \longrightarrow\left[z, z^{\prime}\right] \in S^{3} / \mathbb{Z}_{8}=L_{8} .
$$

The group of natural transformations $\mathrm{O}_{L}$ of this covering contains two elements: the identity and the map $A\left[z, z^{\prime}\right]=\left[\exp (2 \pi i / 8) \cdot z, \exp (2 \pi i / 8) \cdot z^{\prime}\right]$. Now we define the map $f: L_{8} \rightarrow L_{8}$ putting $f\left[z, z^{\prime}\right]=\left[z^{7} /|z|^{6}, z^{\prime 7} /|z|^{\prime 6}\right]$. This map admits the lift $\tilde{f}: L_{4} \rightarrow L_{4}$ given by the same formula and the lift $A \tilde{f}$. We notice that each of the maps $f, \tilde{f}, A \tilde{f}$ is a map of a closed oriented manifold of degree 49 . Since $H_{1}(L ; \mathbb{Q})=H_{2}(L ; \mathbb{Q})=0$ for all lens spaces, the Lefschetz number of each of these three maps equals; $L(f)=1-49=-48 \neq 0$. On the other hand since the lens spaces are Jiang [3], all involved Reidemeister classes are essential hence the Nielsen number equals the Reidemeister number in each case.

Now

$$
\mathscr{R}(f)=\operatorname{coker}(\mathrm{id}-7 \cdot \mathrm{id})=\operatorname{coker}(-6 \cdot \mathrm{id})=\operatorname{coker}(2 \cdot \mathrm{id})=\mathbb{Z}_{2} .
$$

Similarly $\mathscr{R}(\tilde{f})=\mathbb{Z}_{2}$ and $\mathscr{R}(A \cdot \tilde{f})=\mathscr{R}(\tilde{f})=\mathbb{Z}_{2}$ since $A$ is homotopic to the identity. Thus

$$
R(f)=2 \neq 2+2=R(\tilde{f})+R(A \cdot \tilde{f}) .
$$

Since all the classes are essential, the same inequality holds for the Nielsen numbers.

(4) If the group $\left\{\alpha \in \pi_{1}(X ; x) / H(x) ; f_{\#} \alpha=\alpha\right\}$ is trivial for each $x \in \operatorname{Fix}(f)$ lying in an essential Nielsen class of $f$ then all the numbers $I_{i}=J_{i}=1$ and the sum formula holds.

(5) If $\pi_{1} X / H$ is abelian then the rank of the groups

$$
C\left(f_{H \#}\right)=\left\{\alpha \in \pi_{1}(X, x) / H(x) ; f_{\#} \alpha=\alpha\right\}=\operatorname{ker}\left(\mathrm{id}-f_{\#}\right): \pi_{1}(X, x) / H(x) \longrightarrow \pi_{1}(X, x) / H(x)
$$

does not depend on $x \in \operatorname{Fix}(f)$ hence $I$ is constant. If moreover $\pi_{1} X$ is abelian then also the group $C\left(f_{\#}\right)=\operatorname{ker}\left(\mathrm{id}-f_{\#}\right)$ does not depend on $x \in \operatorname{Fix}(f)$. Then we get

$$
N(f)=J / I \cdot\left(N\left(\tilde{f}_{1}\right)+\cdots+N\left(\tilde{f}_{s}\right)\right) .
$$

\section{References}

[1] R. F. Brown, The Nielsen number of a fibre map, Annals of Mathematics. Second Series 85 (1967), 483-493.

[2] _ The Lefschetz Fixed Point Theorem, Scott, Foresman, Illinois, 1971.

[3] B. J. Jiang, Lectures on Nielsen Fixed Point Theory, Contemporary Mathematics, vol. 14, American Mathematical Society, Rhode Island, 1983.

[4] C. Y. You, Fixed point classes of a fiber map, Pacific Journal of Mathematics 100 (1982), no. 1, $217-241$.

Jerzy Jezierski: Department of Mathematics, University of Agriculture, Nowoursynowska 159,

02766 Warszawa, Poland

E-mail address: jezierski_acn@waw.pl 